\title{
New clinical manifestations of COVID-19 related Guillain Barre syndrome higly responsive to intravenous immunoglobulins: two italian cases
}

Andrea Assini

Ospedale Galliera

Luana Benedetti

IRCCS Ospedale Policlinico San Martino

Di Maio Silvia

Intensive Care Unit, Galliera Hospital, Genova, Italy

Schirinzi Erika

Neurology Unit, Galliera Hospital Genova, Italy

Massimo Del Sette ( $\square$ massimo.del.sette@galliera.it )

Neurology Unit, Galliera Hospital Genova, Italy

\section{Short Report}

Keywords: COVID 19, Guillain Barré syndrome, AMSAN, polyneuropathy, Miller Fisher syndrome

Posted Date: May 21st, 2020

DOI: https://doi.org/10.21203/rs.3.rs-30354/v1

License: (c) (1) This work is licensed under a Creative Commons Attribution 4.0 International License. Read Full License 


\section{Abstract}

Recently, some cases of Guillain Barré syndrome (GBS) and Miller Fisher (MF) have been reported, following COVID-19 infection ${ }^{1-2-3}$. We report two different clinical manifestation of Covid-19 related GBS, one is a GBS/MF overlap syndrome, the other one an Acute Motor Sensory Axonal Neuropathy (AMSAN) with massive vegetative impairment, both highly responsive to intravenous immunoglobulins.

\section{Cases}

Case 1

On March, 2020, a 55-year-old man was hospitalized for severe respiratory syndrome preceded by anosmia and ageusia, fever and cough, proven to be due to COVID-19 infection. Laboratory test on admission showed lymphocytopenia associated to the increase of inflammation biomarkers such as PRC, ferritin, LDH. Oropharingeal swab test searching for SARS-CoV-2 was positive. A therapy with idrossichlorochine, arbidol, ritonavir, and lopinavir was promptly started but for the worsening of respiratory function, after three days, the patient was moved to intensive care unit for invasive ventilation. After 20 days from admission the patient showed acute onset of bilateral eyelid ptosis, dysphagia, dysphonia. Neurological examination showed bilateral masseter weakness, tongue protrusion deficit due to bilateral paralysis of the hypoglossal nerve, hyporeflexia of upper and lower limbs, without muscle weakness. Segmental coordination and cognitive functions were normal, too. Speech therapist evaluation showed deficit of elevation of the soft palate due to bilateral 10th cranial nerve palsy. Brain MRI did not show any brain stem lesion. Cerebro Spinal Fluid (CSF) and serum examination showed presence of oligoclonal bands both in CSF and serum, with increased IgG/albumin ratio in CSF (233); total protein level in CSF was normal, yet the patient had low serum albumin level $(2.9 \mathrm{mg} / \mathrm{dl})$. Search for coronavirus in CSF by means of polymerase chain reaction assay was negative. Electroeurography at four limbs revealed a symmetric demyelinating findings and a typical sural sparing pattern. Repetitive nerve stimulation did not show any decremental or incremental pattern as observed in myastenia or myasthenic syndrome. The search for serum anti-ganglioside antibodies was negative. Anticholinergic receptor antibodies were negative. The patient was therefore administered intravenous immunoglobulins ( $0.4 \mathrm{gr} / \mathrm{kG} /$ day for 5 days), with very rapid clinical response on swallowing, speech and tongue motility and strenght, as well as on eyelid ptosis. The first clinical improvements occurred during the fifth day of treatment, with progressively improving trend and complete remission on swallowing and feeding.

\section{Case 2}

A 60-year-old man was hospitalized for fever and cough on March 2020. Blood tests showed lymphocytopenia, increased LDH and GGT, leukocytosis. The patient performed pharyngeal nose swab and thoracic CT scan, positive for severe Covid-related interstitial pneumonia. 3 days after, he showed 
worsening of respiratory functions with the need for tracheostomy and assisted ventilation. He was treated with hydroxychloroquine, antiretroviral therapy and tocilizumab. Over the following days, respiratory symptoms improved with partial recovery of spontaneous breathing. However, 20 days after, the patient presented acute weakness in lower limbs with distal distribution and foot-drop on the right side. Simultaneously, massive disorders of the vegetative nervous system, consisting of gastroplegia, paralytic ileus, loss of blood pressure control occurred. Neurological examination showed distal weakness at four limbs, with foot-drop. Deep tendon reflexes were absent. Electroneurography showed severe sensory-motor axonal polyneuropathy with relative sparing of conduction velocities. The amplitudes of sensory and motor action potentials were significantly reduced. The EMG showed neurogenic changes on the muscles of the 4 limbs, without myogenic pattern. CSF and serum examination showed presence of oligoclonal bands both in CSF and serum, with increased ratio IgG/albumin in CSF (170); total protein level in CSF was normal, yet the patient had low serum albumin level $(2.6 \mathrm{mg} / \mathrm{dl})$ : Direct research for the RNA virus on CSF was negative. The search for anti-ganglioside antibodies was negative. 3 days after the onset of symptoms, intravenous immunoglobulin therapy was started at the standard dosage of $0.4 \mathrm{~g} / \mathrm{kg} /$ day. After 5 days, the vegetative symptomatology significantly improved, with the remission of gastroplegia and recovery of intestinal functions. A subsequent neurological evaluation showed persistence of osteotendinous hyporeflexia but slight improvement in the right foot drop.

In conclusion, we present two cases of Guillain Barre Syndrome, with typical onset latency after SARSCov2 infection. In our Hospital we did not experience an increase in number of GBS cases compared to the same period of the previous year. However, the two cases described fall into the context of peripheral neuropathy related to new coronavirus infection, yet with two very atypical features. In the first case, the impairment of several cranial nerves in association with a demyelinating peripheral neuropathy, suggests an overlap of Miller-Fisher and GBS. Several variants of Guillain-Barré syndrome with involvement of cranial nerves have been described. However, multiple involvement of cranial nerves without motor deficit in limbs is extremely rare, affecting only $5 \%$ of all the cases reported in the literature ${ }^{4-5}$. Recently, Gutierrez-Ortiz and coworkers reported two cases of Miller Fisher syndrome and polyneuritis cranialis in COVID 19, yet they did not perform MRI and neurophysiology ${ }^{2}$. In our patient, the occurrence of cranial nerves I -III-V (motor branch)- IX-X-XII, in association with limbs areflexia and neurophysiological demonstration of demyelinating polyneuropathy, with normal CSF, suggest an overlap between Miller Fisher and GBS.

In the second case, the massive impairment of the vegetative nervous system, associated with electrophysiological changes typical for Acute Motor Sensory Axonal Neuropathy (AMSAN) should be emphasized. Although the association between GBS and vegetative symptoms is described, this is the first case of Covid-related AMSAN, associated with massive vegetative involvement. As suggested by the work of Toscano and coworkers ${ }^{1}$, this case suggest a carefull differential diagnosis between AMSAN and Critical IIIness neuropathy. However, the absence of myogenic pattern on EMG, the significant vegetative 
impairment and the excellent response to immunoglobulins, appear highly suggestive for AMSAN. The normality of proteins in CSF might be justified by the earliness of the examination. Finally, in both cases, the mirror pattern with increase of Ig both in CSF and serum confirms the autoimmune and inflammatory etiology. Unlike the cases with early parainfectious onset reported by others1-2-3, our patients showed the typical latency of GBS symptoms, while an earlier onset of GBS is quite unfrequent. Moreover, the absence of COVID in CSF suggests an immuno-mediated inflammatory mechanism.

In conclusion, these are two cases of polyradicoloneuritis COVID-realted, with very atypical features. Our two cases should be considered variants of GBS (the first in overlap with Miller Fisher, the second in association with severe autonomic neuropathy). Both cases presented an excellent response to immunoglobulin treatment, suggesting the immune-mediated nature of neuropathy

\section{References}

1. Toscano G, Palmerini F, Ravaglia, S, et al.Guillain-Barré Syndrome Associated with SARS-CoV-2 N EnglJ 2000 DOI: 10.1056/NEJMc2009191 Lyu RK, Chen ST.

2. Gutierrez-Ortiz C, Méndez A, Rodrigo-Rey S, et al. Miller Fisher Syndrome and polyneuritis cranialis in COVID-19. Neurology 2000 Published Ahead of Print on April 17, 2020.

3. Ottaviani, Boso F, Tranquillini E, Gapeni I, Pedrotti G, Cozzio S, Guarrera GM, Giometto B.Neurol Sci. 2020 May Early Guillain-Barré syndrome in coronavirus disease 2019 (COVID-19): a case report from an Italian COVID-hospital.

4. Lyu RK, Chen ST. Acute multiple cranial neuropathy: a variant of Guillain-Barré syndrome? Muscle Nerve. 2004 Oct;30(4):433-6.

5. Wang Q, Xiang Y, Yu K, Li C, Wang J, Xiao L. Multiple cranial neuropathy variant of Guillain-Barré syndrome: a case series. Muscle Nerve. 2011 Aug;44(2):252-7. doi: 10.1002/mus.22043.

\section{Declarations}

The study was approved by the local ethic committee

The patients gave informed consent and approved the publication of the manuscript

The authors declare no conflict of interest

The Authors had read and approved the manuscript 